\title{
Utilising crowdsourcing method through BetterCity mobile apps: a case of Tampin district community
}

\begin{abstract}
Nowadays, crowdsourcing has become the hype in knowledge acquisition and diffusion. It is a powerful method that promotes speedy information gathering and at the same time yields better results compared to other similar methods. Increasingly, crowdsourcing has also been actively used for reaching solutions to complex problems. Based on these premises, as well as to demonstrate our social responsibility towards the surrounding community, this paper aims to put fourth our effort through BetterCity while exploiting the advantages of crowdsourcing. It is a mobile application for iOS, which acts as an intermediary between local authority and residents specifically in issues reporting and handling. BetterCity makes the flow of issues reporting easier, achieves complete details of public report, allows the public to contribute information, and most importantly, creates a sense of community by cultivating responsible people to their local areas, together with the local authority.
\end{abstract}

Keyword: Crowdsourcing; Mobile application; Social community 